\title{
Disruption of the Oral Microbiota Is Associated With a Higher Risk of Relapse After Allogeneic Hematopoietic Stem Cell Transplantation
}

Vinícius Campos de Molla ( $\nabla$ viniciuscamposdemolla@gmail.com )

Hospital Sírio-Libanês

Vitor Heidrich

Hospital Sírio-Libanês

Julia Bruno

Hospital Sírio-Libanês

Franciele Knebel

Hospital Sírio-Libanês

Wanessa Miranda-Silva

Hospital Sírio-Libanês

Paula Asprino

Hospital Sírio-Libanês

Luciana Tucunduva

Hospital Sírio-Libanês

Vanderson Rocha

Universidade de São Paulo

Yana Novis

Hospital Sírio-Libanês

Anamaria Camargo

Hospital Sírio-Libanês

Eduardo Fregnani

Hospital Sírio-Libanês

Celso Arrais-Rodrigues

Hospital Sírio-Libanês

\section{Research Article}

Keywords: Oral microbiota, relapse, overall survival, allogeneic hematopoietic stem cell transplantation

Posted Date: May 19th, 2021

DOl: https://doi.org/10.21203/rs.3.rs-512884/v1 
License: (c) (i) This work is licensed under a Creative Commons Attribution 4.0 International License. Read Full License

Version of Record: A version of this preprint was published at Scientific Reports on September 2nd, 2021. See the published version at https://doi.org/10.1038/s41598-021-96939-8. 


\section{Abstract}

Background: Intestinal microbiota (IM) diversity and composition regulates host immunity and affects outcomes after allogeneic stem cell transplantation (allo-HSCT).

Methods: We evaluated how the oral mucosa microbiota (OM) impacts the outcomes in patients who underwent allo-HSCT. Samples from the oral mucosa of 30 patients were collected at three time points: before the conditioning, at aplasia, and at engraftment. We analyzed the associations of OM diversity and composition with allo-HSCT outcomes.

Results: Higher OM diversity at preconditioning was associated with a lower risk of relapse in 3 years ( $33 \%$ versus $68 \%$, respectively; $P=0.04$ ). Dominance (relative abundance $\geq 30 \%$ ) by a single genus at preconditioning was associated with a higher risk of relapse ( $63 \%$ versus $36 \%$, respectively; $P=0.04)$, worse progression-free survival (PFS; $19 \%$ versus $55 \%$, respectively; $P=0.01$ ), and poorer overall survival $(\mathrm{OS})$ at 3 years ( $38 \%$ versus $81 \%$, respectively; $P=0.02$ ).

Conclusion: The presence of Solobacterium at preconditioning was associated with a lower risk of relapse ( $9 \%$ versus $56 \%$, respectively; $P=0.04)$. This is the first study to address the relationship between $\mathrm{OM}$ and clinical outcomes after allo-HSCT.

\section{Highlights}

- Higher OM diversity and the presence of Solobacterium were associated with a lower risk of relapse after allo-HSCT.

- Dominance by a single genus was associated with poor outcomes after allo-HSCT.

\section{Background}

Allogeneic hematopoietic stem cell transplantation (allo-HSCT) remains the only therapeutic option for several hematological neoplasms ${ }^{1}$. Although transplant outcomes have markedly improved in recent decades, relapse of the underlying condition remains the leading cause of death after allo-HSCT ${ }^{2}$. Despite conflicting results, several risk factors have been shown to affect relapse, including the intensity of the conditioning regimen ${ }^{3-5}$, pre-HSCT disease status ${ }^{6}$, donor age ${ }^{7,8}$, graft source ${ }^{9}$, killer immunoglobulin-like receptor compatibility ${ }^{10}$, graft versus host disease (GVHD) prophylaxis ${ }^{11-13}$, and the occurrence of chronic GVHD (cGVHD) ${ }^{14}$. Infections, acute GVHD (aGVHD), cGVHD, and secondary neoplasia are the main causes of non-relapse mortality (NRM) ${ }^{2,15}$. The disease risk index (DRI) stratifies the risk of mortality in patients after allo-HSCT, according to diagnosis and disease status ${ }^{16}$.

The intestinal microbiota (IM) has been shown to play a vital role in regulating host immunity ${ }^{17}$ and improving antineoplastic activity ${ }^{18,19}$. In addition, IM disruption, characterized by significant changes in microbiota diversity and composition, is associated with allo-HSCT clinical outcomes. Common 
complications after allo-HSCT, such as infections, mucositis, and GVHD, are associated with significant changes in IM diversity and composition. In allo-HSCT, IM disruption is also associated with the incidence of $\mathrm{GVHD}^{20-22}$, overall survival $(\mathrm{OS})^{23-26}$, and underlying disease relapse 27,28 .

The human oral cavity harbors the second most abundant microbiota after the gastrointestinal tract. As observed for the IM, the oral microbiota (OM) directly influences human health ${ }^{29}$. OM disruption has been observed in several diseases, including diabetes, autoimmune diseases, endocarditis, gastrointestinal cancer, head and neck cancer ${ }^{30-32}$, and acute lymphoblastic leukemia ${ }^{33}$. Changes in the OM in patients undergoing allo-HSCT are known to be associated with respiratory signs and symptoms ${ }^{34}$ and oral mucositis ${ }^{35}$; however, no correlation between OM and allo-HSCT outcomes have been reported to date.

Accordingly, in this study, we evaluated whether the OM disruption is related to outcomes in patients who underwent allo-HSCT.

\section{Methods}

\section{Patient characteristics and sample collection}

We collected samples from the oral mucosa of patients who underwent allo-HSCT at Hospital Sírio Libanês, São Paulo, Brazil between January 2016 and April 2018.

Samples were collected by rubbing the dorsal tongue and buccal and alveolar mucosa with sterile swabs at three time points: before the conditioning regimen (preconditioning), at aplasia (defined as the first day of neutrophils under $0.5 \times 10^{3} / \mathrm{uL}$ ), and at engraftment. All patients were requested not to perform oral hygiene for at least $6 \mathrm{~h}$ before collection. Informed consent was obtained from all patients before collection. The study was approved by the local ethics committee (Comitê de Ética e Pesquisa - Hospital Sírio Libanês), according to the Declaration of Helsinki. No tissue was procured from prisoners in this study. All patients were examined by an oral medicine specialist for potential infections, and all followed the same protocol for oral mucositis prophylaxis with photobiomodulation and oral hygiene with fluoride toothpaste and $0.12 \%$ chlorhexidine mouthwash. The standard antimicrobial prophylaxis in our institution included oral levofloxacin, acyclovir, and antifungal prophylaxis according to the patient's risk of fungal infection (voriconazole for high-risk patients, and fluconazole for low risk patients).

\section{DNA extraction}

Bacterial cells were recovered from oral mucosa swabs through vortexing in TE buffer supplemented with $6 \mu \mathrm{L}$ PureLink RNAse A (20 mg/mL; Thermo Fisher Scientific, Waltham, MA, USA). DNA was extracted using a QIAamp DNA Blood Mini Kit (Qiagen, Hilden, Germany) according to the manufacturer's protocol (DNA Purification from Blood or Body Fluids) and stored at $-80^{\circ} \mathrm{C}$. 


\section{S rRNA amplicon sequencing}

For $16 \mathrm{~S}$ rRNA amplicon sequencing, $12.5 \mathrm{ng}$ DNA and prevalidated primers ${ }^{36}$ were used to amplify $16 \mathrm{~S}$ rRNA hypervariable regions V3-V4. Amplicons were sequenced as described elsewhere ${ }^{37}$ on an Illumina MiSeq platform (Illumina, San Diego, CA, USA).

\section{Bioinformatics pipeline}

Reads were demultiplexed, and primer sequences were removed using the MiSeq Reporter software. Within the QIIME 2 framework ${ }^{38}$, using experiment-specific adaptive error models ${ }^{39}$, forward and reverse sequences were filtered for quality and bimeras, denoised, and merged into consensus sequences with the DADA2 pipeline ${ }^{40}$, generating unique amplicon sequencing variants (ASVs). ASVs were further filtered for chimeric sequences using the SILVA database ${ }^{41}$ and UCHIME ${ }^{42}$. ASVs were taxonomically assigned using SILVA database and VSEARCH tool ${ }^{43}$.

\section{Statistical analyses}

For alpha diversity analyses, the samples were rarefied to 12,500 reads before calculating the Shannon index, Simpson index, or the number of observed ASVs as bacterial diversity measures with the QIIME 2 q2-diversity plugin. Alpha diversity across groups was compared with the Mann-Whitney U test. OM was classified as having high or low diversity based on the median Shannon index diversity measure across the study population at a given collection time point. Fisher's exact tests and two-sided Student's t-tests were used to evaluate the associations between alpha diversity status and categorical and numerical clinical parameters, respectively. The relative abundance of each taxa was calculated with the QIIME 2 q2-taxa plugin. The taxa shown on relative abundance longitudinal plots are all those showing dominance (relative abundance $\geq 30 \%$ ) in at least one study sample or relative abundance $\geq 5 \%$ in at least $25 \%$ of study samples. Differentially abundant genera across transplantation phases were identified using ANCOM test, with relative differences represented by the log-transformed average relative abundance fold change between groups. ANCOM W represents the proportion of null hypotheses rejected when sub-testing the differential abundance of a genus normalized by the abundance of each one of the genera in the dataset. $\mathrm{W}>0.7$ was considered as statistically significant. The relative abundance of a genus was considered to increase during allo-HSCT for a given patient when the relative abundance at engraftment was greater than at preconditioning and the final relative abundance was $\geq 0.1 \%$. The probabilities of progression-free survival (PFS) and OS were calculated using the Kaplan-Meier method and compared using log-rank tests. Cumulative incidence rates were calculated for aGVHD, cGVHD, NRM, and relapse/progression, with death being considered a competing event. Ninety-five percent confidence intervals ( $95 \% \mathrm{Cls}$ ) were estimated using the Greenwood formula. Adjusted probabilities for outcomes after transplantation were estimated using the Cox proportional hazards method (PFS and OS) and FineGray risk regression model (aGVHD, cGVHD, NRM, and relapse/progression). The association between 
OM parameters and HSCT outcome was investigated in the final model after adjusting for the DRI. Firstorder interactions between $\mathrm{OM}$ parameters and each variable of interest were examined. The results are presented as relative risks of failure (adverse prognostic factors versus good prognostic factors), with 95\% Cls and two-tailed $P$ values. To examine the association between genus presence at preconditioning and relapse, only genera present in $25-75 \%$ of samples were evaluated, where presence was defined as relative abundance $\geq 0.1 \%$. R software (version 3.5.0) and RStudio (version 1.2.5033) were used for statistical analyses. The statistical package cmprsk was used to evaluate relapse across groups with transplant-related death as the competing risk.

\section{Results}

\section{Patient characteristics}

Between January 2016 and April 2018, 30 patients who underwent allo-HSCT for hematologic malignancies and had oral mucosa samples collected were included in this study. The most common underlying diseases were acute myeloid leukemia and acute lymphoblastic leukemia (60\%). Conditioning regimens and intensity, graft source, T-cell depletion, and other clinical characteristics are listed in Table 1. The median follow-up time for survivors was 41 (30-50) months.

\section{Microbiota dynamics analyses}

In total, 5,920,836 high-quality bacterial assigned sequencing reads were analyzed, representing 1723 unique ASVs. Out of the 90 samples sequenced, nine were excluded from diversity analyses owing to an insufficient number of high-quality reads $(<12,500$ reads per sample, as determined using alpha diversity rarefaction curves) after the read-filtering steps employed in the pipeline. Therefore, adequate preconditioning samples were available for 27 of the 30 patients included in this study.

The intrasample bacterial diversity (Figure 1A) and richness (Figure S1) of OM samples decreased significantly during the clinical course. This drop in diversity is associated with changes in taxa relative abundance during the same period (Figure S2). Notably, all patients showed bacterial dominance by a single genus after preconditioning. In Figure 1B, we show three representative patients with major dominance (relative abundance $>80 \%$ ) by a single genus (Stenotrophomonas, Rothia, and Veillonella, respectively) at engraftment.

For a broader assessment of the relative abundance changes from preconditioning to subsequent transplantation phases, we employed the ANCOM test at the genus level. We observed statistically significant variations in the abundance of both opportunistic pathogenic and commensal genera (Figure S3). From preconditioning to aplasia, there was a significant increase in the abundance of the potentially pathogenic genera Enterococcus and Lactobacillus, which were even more increased in the engraftment phase in terms of relative abundance fold change from preconditioning. Staphylococcus and Mycoplasma were other potentially pathogenic genera increased at engraftment. Contrarily, there was a 
significant decrease in the abundance of the commensal genera Haemophilus (at aplasia) and Gemella (at engraftment).

A global increase of potentially pathogenic genera occurs during allo-HSCT. However, evaluating each patient individually, we noticed irregular changes in the relative abundance of those same genera from preconditioning to engraftment. An increase in the relative abundance of Enterococcus, Lactobacillus, Staphylococcus, and Mycoplasma was observed in $32 \%, 40 \%, 56 \%$, and $68 \%$ of patients (Figure S4). Patients who presented an increase in Enterococcus relative abundance had a higher incidence of cGVHD when compared with patients without the increase of relative abundance $(P=0.03)$. No other associations between the increase in the relative abundance of potentially pathogenic genera and alloHSCT outcomes was observed (Table S1).

\section{Impact of OM diversity on transplant outcomes}

In order to elucidate the impact of OM bacterial diversity on allo-HSCT outcomes, we stratified patients into low or high diversity at each collection time (Table S2). A swimmer plot was used to illustrate these correlations at preconditioning (Figure 2A). When we compared those with high or low OM diversity at preconditioning, no differences were found in PFS (36\% versus $32 \%$, respectively; hazard ratio $[\mathrm{HR}]=0.75$, $95 \% \mathrm{Cl}: 0.28-2.00, P=0.57)$, or in OS at 3 years ( $54 \%$ versus $57 \%$, respectively; $\mathrm{HR}=0.96,95 \% \mathrm{Cl}$ : $0.33-$ $2.89, P=0.96)$. We also did not observe any differences in aGVHD at 100 days $(43 \%$ versus $62 \%$, respectively; $\mathrm{HR}=1.77,95 \% \mathrm{Cl}: 0.66-4.81, P=0.26)$ or cGVHD at 3 years ( $30 \%$ versus $7 \%$, respectively; $\mathrm{HR}=4.79,95 \% \mathrm{Cl}: 0.56-40.8, P=0.15)$. However, high OM diversity at preconditioning was associated with a lower risk of relapse at 3 years when compared with low diversity (33\% versus $68 \%$, respectively; $\mathrm{HR}=0.27,95 \% \mathrm{Cl}: 0.07-0.97, P=0.04$; Figure 2B, Table S3).

Notably, 16 (59\%) patients presented some type of bacterial dominance at preconditioning. Such events encompassed 4 different genera, all of which are oral commensal: Streptococcus (dominant in 9/16 patients) and Veillonella (dominant in 2/16 patients), both members of the Firmicutes phylum; Neisseria (dominant in 3/16 patients) and Rothia (dominant in 2/16 patients). Genus dominance was detected even among patients classified as having high diversity at preconditioning (Figure $2 \mathrm{~A}$ ). The presence of dominance by any genus at preconditioning was also associated with an increased risk of relapse at 3 years when compared with the absence of dominance $(63 \%$ versus $36 \%$, respectively; $\mathrm{HR}=4.59,95 \% \mathrm{Cl}$ : $1.11-19, P=0.03$; Figure $3 \mathrm{~A}$ ). When evaluating dominance by specific genera or types of genera at preconditioning, neither dominance by Streptococcus (56\% versus $39 \%$, respectively; $\mathrm{HR}=1.64,95 \% \mathrm{Cl}$ : $0.52-5.14, P=0.4$ ), nor dominance by facultative anaerobic genera (Streptococcus or Rothia; $56 \%$ versus $39 \%$, respectively; $\mathrm{HR}=2.05,95 \% \mathrm{Cl}: 0.67-6.27, P=0.21$ ) were associated with an increased risk of relapse. Due to the very unequal group sizes, we could not evaluate the association between dominance by Rothia (2/27 patients), Veillonella (the only dominant anaerobe; $2 / 27$ patients) or Neisseria (the only dominant aerobe; $3 / 27$ patients) at preconditioning and the risk of relapse. 
Additionally, the presence of dominance by any genus at preconditioning was associated with inferior PFS (19\% versus 55\%, respectively; $\mathrm{HR}=4.75,95 \% \mathrm{Cl}: 1.78-12.7, P=0.01$; Figure $3 \mathrm{~B})$ and $\mathrm{OS}(38 \%$ versus $81 \%$, respectively; $\mathrm{HR}=4.73,95 \% \mathrm{Cl}: 1.59-14.08, P=0.02$; Figure $3 \mathrm{C})$. No differences in aGVHD at 100 days (43\% versus $63 \%$, respectively; $\mathrm{HR}=0.50,95 \% \mathrm{Cl}: 0.18-1.37, P=0.18)$, cGVHD at 3 years $(19 \%$ versus $18 \%$, respectively; $\mathrm{HR}=1.07,95 \% \mathrm{Cl}: 0.19-5.93, P=0.94)$, or NRM at 3 years $(20 \%$ versus $9 \%$, respectively; $\mathrm{HR}=2.35,95 \% \mathrm{Cl}: 0.27-20.60, P=0.44)$ were observed.

As expected, we also observed that patients with a high DRI had a significantly higher risk of relapse/progression, as compared with those with low-intermediate DRI at 3 years ( $62 \%$ versus $12 \%$, respectively; $\mathrm{HR}=10.2,95 \% \mathrm{Cl}: 2.24-46.7, P<0.01)$ and worse OS $(77 \%$ versus $30 \%$, respectively; $\mathrm{HR}=$ $4.07,95 \% \mathrm{Cl}: 1.38-11.97, P=0.01)$.

After adjusting analyses for the DRI, there was a trend toward a higher risk of relapse/progression in those with low $\mathrm{OM}$ diversity $(\mathrm{HR}=0.30,95 \% \mathrm{Cl}$ : $0.08-1.09, P=0.07$; Figure $2 \mathrm{C})$, and bacterial dominance of any genus remained significantly associated with the risk of relapse $(\mathrm{HR}=4.19,95 \% \mathrm{Cl}$ : 1.25-14.1, $P=$ 0.02; Figure 3D) and worse PFS (HR $=4.14,95 \% \mathrm{Cl}: 1.15-14.89, P=0.03$; Figure 3E); there was a tendency for bacterial dominance of any genus to also be related to worse OS ( $\mathrm{HR}=4.12,95 \% \mathrm{Cl}$ : $0.89-$ $19.13, P=0.07$; Figure 3F).

Other relevant clinical parameters, such as conditioning intensity, underlying disease, and graft source, were not significantly associated with the risk of relapse (Figure S5, Table S4).

\section{Genus presence and transplant outcomes}

As the genus level represents the most specific taxonomic level that still provides reliable taxonomic classification for V3-V4 amplicons, to further evaluate the association between preconditioning $\mathrm{OM}$ and transplant outcomes, we analyzed whether any non-core genus (those present in $25-75 \%$ of samples) was associated with a higher risk of relapse. In this exploratory analysis (without adjustment for multiple comparisons), of the 18 genera that matched the selection criteria tested in a univariate analysis for relapse (Figure 4A, Figure S6), only Solobacterium was significantly associated with lower relapse risk ( $9 \%$ versus $56 \%$, respectively; $\mathrm{HR}=0.23,95 \% \mathrm{Cl}: 0.05-0.94, P=0.04$; Figure $4 \mathrm{~B}$ ), and this association remained significant after adjusting for $\mathrm{DRI}(\mathrm{HR}=0.20,95 \% \mathrm{Cl}: 0.06-0.67, P=0.01$; Figure $4 \mathrm{C})$. However, after adjusting for multiple comparisons using the Bonferroni correction, because of the limited statistical power of this study, the univariate association between Solobacterium presence and lower relapse risk loses significance $(P=0.72)$. The relative abundance of Solobacterium at preconditioning per patient is depicted in Figure S7. No differences in the presence of Solobacterium were found in other outcomes (aGVHD at 100 days: $64 \%$ versus $44 \%$, respectively [HR $=1.84,95 \% \mathrm{Cl}: 0.68-4.95, P=0.23]$; CGVHD: $27 \%$ versus $13 \%$, respectively $[\mathrm{HR}=2.41,95 \% \mathrm{Cl}: 0.43-13.4, P=0.31]$; PFS: $55 \%$ versus $37 \%$, respectively $[\mathrm{HR}=$ $0.83,95 \% \mathrm{Cl}: 0.31-0.83, P=0.71]$; and OS at 3 years: $55 \%$ versus $28 \%$, respectively [HR $=0.99,95 \% \mathrm{Cl}$ : $0.32-3.08, P=0.99])$. 


\section{Antibiotic usage}

From 1 week before the start of the conditioning regimen to engraftment, 28 (93\%) patients used antibiotics to treat febrile neutropenia; 20 (67\%) used cefepime, 16 (53\%) used meropenem, and four (13\%) used piperacillin-tazobactam. None of these antibiotics were associated with the risk of relapse (Figure S5).

We could not analyze the association between the use of antibiotics before transplant ( 30 days before starting the conditioning regimen) and $\mathrm{OM}$ bacterial diversity because of the small number of patients who used antibiotics at that time point.

\section{Discussion}

In this single-center observational study, we prospectively collected samples from the oral mucosa of patients who underwent allo-HSCT and observed that patients who presented low OM diversity or dominance of any genus before conditioning had a significantly increased risk of relapse. The dominance of any genus was also associated with worse PFS and OS. To the best of our knowledge, this is the first study to evaluate the possible impact of the OM on allo-HSCT outcomes. Additionally, in our study, we used ASVs, which are read sequences denoised to single-nucleotide resolution, a technique with higher sensitivity and specificity and that is more reproducible and comprehensive than operational taxonomic units ${ }^{45-47}$, used in most of the previous studies of the IM.

The $\mathrm{OM}$ has different niches in the same environment and is as diverse as the IM. Previous studies evaluating OM and allo-HSCT have shown conflicting results, likely because of the use of low-resolution techniques for microbiota analysis and the small sample sizes. In one case series, there were no changes in OM after allo-HSCT. The most common oral organisms, e.g., Streptococcus, Gemella, and Veillonella, remained relatively stable after transplant ${ }^{34}$. However, another study showed a reduction in alpha diversity after allo-HSCT when compared with the pretransplant $\mathrm{OM}^{35}$, and this reduction was more pronounced in patients who developed oral mucositis ${ }^{48}$. Besides, we did not find any direct correlation between the use of antibiotics after conditioning and transplant outcomes, as other studies have shown for IM diversity 23,24 .

Recently, IM has attracted attention as a potential predictive marker for allo-HSCT outcomes. Previous studies have shown that low IM diversity is associated with a higher risk of mortality, but not with the risk of relapse ${ }^{25-27}$, diverging from our findings.

Higher risk of aGVHD in patients with low IM diversity ${ }^{49}$ and a higher risk of transplant-related mortality attributable to $\mathrm{GVHD}^{26}$ were also reported. In the oral mucosa samples analyzed in the current study, low OM diversity was associated with an increased risk of relapse but did not change the risk of mortality, aGVHD or CGVHD. 
The dominance of a specific bacterial group in IM, Eubacterium limosum, has also been shown to be related to relapse and disease progression. In our series, the dominance of any genus was associated with a higher risk of relapse.

As opposed to what has been observed for $\mathrm{IM}^{27}$, all dominant genera at preconditioning reported herein are commensal organisms. Thus, it is unlikely that they all have detrimental roles in the allo-HSCT setting, being more plausible that the presence of dominance by any genus is a proxy for low diversity/dysbiotic $\mathrm{OM}$.

Furthermore, the presence of Solobacterium in the OM before conditioning seems to have a protective effect against relapse. S. moorei, the only species in the Solobacterium genus, is normally associated with halitosis ${ }^{50,51}$ and endodontic infection ${ }^{52,53}$. However, in the allo-HSCT scenario, the lack of Solobacterium could be a marker of dysbiosis, pretransplant disease status, or previous treatments. Alternatively, this genus may also play a role as an immune mediator by producing hydrogen sulfide ${ }^{50}$, a metabolite associated with decreased oxidative stress and increased sensitivity to antibiotics ${ }^{54}$. Although, the low overall Solobacterium relative abundance even in patients where it was present makes the latter alternative more unlikely, this finding need to be validated in future studies.

A previous study analyzed the tongue microbiota in patients who underwent alloHSCT and compared it with community-dwelling adults. AlloHSCT patients have a lower tongue microbiota alpha diversity when compared to community adults. Moreover, the presence of Staphylococcus haemolyticus or Ralstonia pickettii was associated with a higher risk of mortality. Nevertheless, no relationship was observed between alpha diversity of the tongue microbiota and incidence of transplant complications ${ }^{47}$. A study of salivary microbiota showed a reduction in alpha diversity during the course of transplantation. Again, no correlation between salivary microbiota diversity and alloHSCT outcomes was found ${ }^{55}$. The discrepancies between these studies and our findings may be related to different sites of sample collections, and different distinct microbiome analysis techniques.

Our study had several limitations of a relatively small and heterogenous single-center transplant cohort. However, as observed in studies of IM, in our series, OM showed a significant correlation with relapse and may also provide valuable information on host-related microbial dysbiosis, providing a simple, reproducible technique for collection and analysis prior to transplantation.

\section{Conclusion}

In the current study, we focused on preconditioning samples in order to identify predictive biomarkers. This was the first study that analyzed the potential clinical effects of OM on allo-HSCT outcomes and as a biomarker for relapse. However, prospective trials and validation cohorts are needed to confirm these findings and to test whether early interventions to correct $\mathrm{OM}$ dysbiosis or more aggressive strategies to prevent relapse in $\mathrm{OM}$ dysbiotic patients, such as early immunosuppression withdrawal, maintenance therapy, or prophylactic donor lymphocyte infusions, could improve allo-HSCT outcomes. 


\section{Abbreviations}

95\% Cis: Ninety-five percent confidence intervals; aGVHD: acute GVHD; allo-HSCT: allogeneic stem cell transplantation; ASVs: amplicon sequencing variants; cGVHD: chronic GVHD; DRI: disease risk index; GVHD: graft versus host disease; IM: Intestinal microbiota; NRM: non-relapse mortality; OM: oral mucosa microbiota; OS: overall survival; PFS: progression-free survival.

\section{Declarations}

\section{ACKNOWLEDGEMENTS}

We are grateful to all patients from Hospital Sírio Libanês, São Paulo, Brazil. We thank our colleagues from Hospital Sírio Libanês.

\section{AUTHORS' CONTRIBUTIONS}

Conception and design: Anamaria Aranha Camargo, Eduardo R. Fregnani, Celso Arrais-Rodrigues

Collection and assembly of data:' Vitor Heidrich, Julia S. Bruno, Franciele H. Knebel, Wanessa MirandaSilva, Paula F. Asprino, Vinícius C. de Molla, Celso Arrais-Rodrigues

Data analysis and interpretation: Vinícius C. de Molla, Vitor Heidrich, Julia S. Bruno, Anamaria Aranha Camargo, Eduardo R. Fregnani, Celso Arrais-Rodrigues

Manuscript writing: All authors

Final approval of manuscript: All authors

Accountable for all aspects of the work: All authors

\section{FUNDING}

V.C.M. was supported by Conselho Nacional de Desenvolvimento Científico e Tecnológico (CNPq process no. 141575/2018-2). J.S.B. was supported by Coordenação de Aperfeiçoamento de pessoal de Nível Superior (CAPES process no. 001). V.H. was supported by Fundação de Amapro à Pesquisa do Estado de São Paulo (FAPESP process no. 13996-0/2018).

\section{Ethics approval and consent to participate}

The study was approved by the local ethics committee, according to the Declaration of Helsinki.

\section{Consent for publication}

Not applicable. 


\section{Competing interests}

The authors declare that they have no competing interests.

\section{References}

1. Gyurkocza B, Sandmaier BM. Conditioning regimens for hematopoietic cell transplantation: one size does not fit all. Blood 2014; 124: 344-353.

2. Styczyński J, Tridello G, Koster L, lacobelli S, van Biezen A, van der Werf $S$ et al. Death after hematopoietic stem cell transplantation: changes over calendar year time, infections and associated factors. Bone Marrow Transplant 2020; 55: 126-136.

3. Ossenkoppele GJ, Janssen JJWM, van de Loosdrecht AA. Risk factors for relapse after allogeneic transplantation in acute myeloid leukemia. Haematologica 2016; 101: 20-25.

4. Martino R, de Wreede L, Fiocco M, van Biezen A, von dem Borne PA, Hamladji R-M et al. Comparison of conditioning regimens of various intensities for allogeneic hematopoietic SCT using HLA-identical sibling donors in AML and MDS with $<10 \%$ BM blasts: a report from EBMT. Bone Marrow Transplant 2013; 48: 761-770.

5. Bornhäuser M, Kienast J, Trenschel R, Burchert A, Hegenbart U, Stadler M et al. Reduced-intensity conditioning versus standard conditioning before allogeneic haemopoietic cell transplantation in patients with acute myeloid leukaemia in first complete remission: a prospective, open-label randomised phase 3 trial. Lancet Oncol 2012; 13: 1035-1044.

6. Shen Z, Gu X, Mao W, Yin L, Yang L, Zhang Z et al. Influence of pre-transplant minimal residual disease on prognosis after Allo-SCT for patients with acute lymphoblastic leukemia: Systematic review and meta-analysis. BMC Cancer 2018; 18: 1-12.

7. Rezvani AR, Storer BE, Guthrie KA, Schoch HG, Maloney DG, Sandmaier BM et al. Impact of Donor Age on Outcome after Allogeneic Hematopoietic Cell Transplantation. Biol Blood Marrow Transplant 2015; 21: 105-112.

8. Bastida JM, Cabrero M, Lopez-Godino O, Lopez-Parra M, Sanchez-Guijo F, Lopez-Corral L et al. Influence of donor age in allogeneic stem cell transplant outcome in acute myeloid leukemia and myelodisplastic syndrome. Leuk Res 2015; 39: 828-834.

9. Milano F, Gooley T, Wood B, Woolfrey A, Flowers ME, Doney K et al. Cord-Blood Transplantation in Patients with Minimal Residual Disease. N Engl J Med 2016; 375: 944-953.

10. Willemze R, Rodrigues CA, Labopin M, Sanz G, Michel G, Socié G et al. KIR-ligand incompatibility in the graft-versus-host direction improves outcomes after umbilical cord blood transplantation for acute leukemia. Leukemia 2009; 23: 492-500.

11. Soiffer RJ, Kim HT, McGuirk J, Horwitz ME, Johnston L, Patnaik MM et al. Prospective, Randomized, Double-Blind, Phase III Clinical Trial of Anti-T-Lymphocyte Globulin to Assess Impact on Chronic Graft-Versus-Host Disease-Free Survival in Patients Undergoing HLA-Matched Unrelated Myeloablative Hematopoietic Cell Transplantation. J Clin Oncol 2017; 35: 4003-4011. 
12. Walker I, Panzarella T, Couban S, Couture F, Devins G, Elemary M et al. Addition of anti-thymocyte globulin to standard graft-versus-host disease prophylaxis versus standard treatment alone in patients with haematological malignancies undergoing transplantation from unrelated donors: final analysis of a randomised, open-label. Lancet Haematol 2020; 7: e100-e111.

13. Nykolyszyn C, Granata A, Pagliardini T, Castagna L, Harbi S, Bouabdallah R et al. Posttransplantation cyclophosphamide vs. antithymocyte globulin as GVHD prophylaxis for mismatched unrelated hematopoietic stem cell transplantation. Bone Marrow Transplant 2020; 55: 349-355.

14. Signori A, Crocchiolo R, Oneto R, Sacchi N, Sormani MP, Fagioli F et al. Chronic GVHD is associated with inferior relapse risk irrespective of stem cell source among patients receiving transplantation from unrelated donors. Bone Marrow Transplant 2012; 47: 1474-1478.

15. Tanaka Y, Kurosawa S, Tajima K, Tanaka T, Ito R, Inoue $Y$ et al. Analysis of non-relapse mortality and causes of death over 15 years following allogeneic hematopoietic stem cell transplantation. Bone Marrow Transplant 2016; 51: 553-559.

16. Armand P, Kim HT, Logan BR, Wang Z, Alyea EP, Kalaycio ME et al. Validation and refinement of the Disease Risk Index for allogeneic stem cell transplantation. Blood 2014; 123: 3664-3671.

17. Belkaid Y, Hand TW. Role of the Microbiota in Immunity and Inflammation. Cell 2014; 157: 121-141.

18. Zitvogel L, Galluzzi L, Viaud S, Vétizou M, Daillère R, Merad M et al. Cancer and the gut microbiota: An unexpected link. Sci Trans/ Med 2015; 7: 271ps1-271ps1.

19. Vetizou M, Pitt JM, Daillere R, Lepage P, Waldschmitt N, Flament C et al. Anticancer immunotherapy by CTLA-4 blockade relies on the gut microbiota. Science (80-) 2015; 350: 1079-1084.

20. Holler E, Butzhammer P, Schmid K, Hundsrucker C, Koestler J, Peter K et al. Metagenomic Analysis of the Stool Microbiome in Patients Receiving Allogeneic Stem Cell Transplantation: Loss of Diversity Is Associated with Use of Systemic Antibiotics and More Pronounced in Gastrointestinal Graft-versusHost Disease. Biol Blood Marrow Transplant 2014; 20: 640-645.

21. Mathewson ND, Jenq R, Mathew A V., Koenigsknecht M, Hanash A, Toubai T et al. Gut microbiomederived metabolites modulate intestinal epithelial cell damage and mitigate graft-versus-host disease. Nat Immunol 2016; 17: 505-513.

22. Eriguchi Y, Takashima S, Oka H, Shimoji S, Nakamura K, Uryu H et al. Graft-versus-host disease disrupts intestinal microbial ecology by inhibiting Paneth cell production of a-defensins. Blood 2012; 120: $223-231$.

23. Jenq RR, Taur Y, Devlin SM, Ponce DM, Goldberg JD, Ahr KF et al. Intestinal Blautia Is Associated with Reduced Death from Graft-versus-Host Disease. Biol Blood Marrow Transplant 2015; 21: 1373-1383.

24. Shono Y, Docampo MD, Peled JU, Perobelli SM, Velardi E, Tsai JJ et al. Increased GVHD-related mortality with broad-spectrum antibiotic use after allogeneic hematopoietic stem cell transplantation in human patients and mice. Sci Transl Med 2016; 8: 339ra71-339ra71.

25. Taur Y, Jenq RR, Perales M-A, Littmann ER, Morjaria S, Ling L et al. The effects of intestinal tract bacterial diversity on mortality following allogeneic hematopoietic stem cell transplantation. Blood 2014; 124: 1174-1182. 
26. Peled JU, Gomes ALC, Devlin SM, Littmann ER, Taur Y, Sung AD et al. Microbiota as Predictor of Mortality in Allogeneic Hematopoietic-Cell Transplantation. N Engl J Med 2020; 382: 822-834.

27. Peled JU, Devlin SM, Staffas A, Lumish M, Khanin R, Littmann ER et al. Intestinal Microbiota and Relapse After Hematopoietic-Cell Transplantation. J Clin Oncol 2017; 35: 1650-1659.

28. Shono Y, Van Den Brink MRM. Gut microbiota injury in allogeneic haematopoietic stem cell transplantation. Nat Rev Cancer 2018; 18: 283-295.

29. Idris A, Hasnain SZ, Huat LZ, Koh D. Human diseases, immunity and the oral microbiota-Insights gained from metagenomic studies. Oral Sci Int 2017; 14: 27-32.

30. Mascitti M, Togni L, Troiano G, Caponio VCA, Gissi DB, Montebugnoli L et al. Beyond Head and Neck Cancer: The Relationship Between Oral Microbiota and Tumour Development in Distant Organs. Front Cell Infect Microbiol 2019; 9. doi:10.3389/fcimb.2019.00232.

31. Zhao H, Chu M, Huang Z, Yang X, Ran S, Hu B et al. Variations in oral microbiota associated with oral cancer. Sci Rep 2017; 7: 11773.

32. Zhang Y, Niu Q, Fan W, Huang F, He H. Oral microbiota and gastrointestinal cancer. Onco Targets Ther 2019; Volume 12: 4721-4728.

33. Wang Y, Xue J, Zhou X, You M, Du Q, Yang X et al. Oral Microbiota Distinguishes Acute Lymphoblastic Leukemia Pediatric Hosts from Healthy Populations. PLoS One 2014; 9: e102116.

34. Ames NJ, Sulima P, Ngo T, Barb J, Munson PJ, Paster BJ et al. A Characterization of the Oral Microbiome in Allogeneic Stem Cell Transplant Patients. PLoS One 2012; 7. doi:10.1371/journal.pone.0047628.

35. Ames NJ, Barb JJ, Ranucci A, Kim H, Mudra SE, Cashion AK et al. The oral microbiome of patients undergoing treatment for severe aplastic anemia: a pilot study. Ann Hematol 2019; 98: 1351-1365.

36. Klindworth A, Pruesse E, Schweer T, Peplies J, Quast C, Horn M et al. Evaluation of general 16S ribosomal RNA gene PCR primers for classical and next-generation sequencing-based diversity studies. Nucleic Acids Res 2013; 41: e1-e1.

37. Wang H, Altemus J, Niazi F, Green H, Calhoun BC, Sturgis C et al. Breast tissue, oral and urinary microbiomes in breast cancer. Oncotarget 2017; 8. doi:10.18632/oncotarget.21490.

38. Bolyen E, Rideout JR, Dillon MR, Bokulich NA, Abnet CC, Al-Ghalith GA et al. Reproducible, interactive, scalable and extensible microbiome data science using QIIME 2. Nat Biotechnol 2019; 37: 852-857.

39. Edgar RC, Flyvbjerg $\mathrm{H}$. Error filtering, pair assembly and error correction for next-generation sequencing reads. Bioinformatics 2015; 31: 3476-3482.

40. Callahan BJ, McMurdie PJ, Rosen MJ, Han AW, Johnson AJA, Holmes SP. DADA2: High-resolution sample inference from Illumina amplicon data. Nat Methods 2016; 13: 581-583.

41. Quast C, Pruesse E, Yilmaz P, Gerken J, Schweer T, Yarza P et al. The SILVA ribosomal RNA gene database project: improved data processing and web-based tools. Nucleic Acids Res 2012; 41: D590-D596. 
42. Edgar RC, Haas BJ, Clemente JC, Quince C, Knight R. UCHIME improves sensitivity and speed of chimera detection. Bioinformatics 2011; 27: 2194-2200.

43. Rognes T, Flouri T, Nichols B, Quince C, Mahé F. VSEARCH: a versatile open source tool for metagenomics. PeerJ 2016; 4: e2584.

44. Kaul A, Mandal S, Davidov O, Peddada SD. Analysis of Microbiome Data in the Presence of Excess Zeros. Front Microbiol 2017; 8: 1-10.

45. Edgar RC. UPARSE: highly accurate OTU sequences from microbial amplicon reads. Nat Methods 2013; 10: 996-998.

46. Callahan BJ, McMurdie PJ, Holmes SP. Exact sequence variants should replace operational taxonomic units in marker-gene data analysis. ISME J 2017; 11: 2639-2643.

47. Oku S, Takeshita T, Futatsuki T, Kageyama S, Asakawa M, Mori Y et al. Disrupted tongue microbiota and detection of nonindigenous bacteria on the day of allogeneic hematopoietic stem cell transplantation. PLOS Pathog 2020; 16: e1008348.

48. Shouval R, Eshel A, Danylesko I, Dubovski B, Fein JA, Fried S et al. Salivary Microbial and Metabolic Determinants of Oral Mucositis in Recipients of Allogeneic Hematopoietic Stem Cell Transplantation. Biol Blood Marrow Transplant 2020; 26: S50.

49. Golob JL, Pergam SA, Srinivasan S, Fiedler TL, Liu C, Garcia K et al. Stool Microbiota at Neutrophil Recovery Is Predictive for Severe Acute Graft vs Host Disease After Hematopoietic Cell Transplantation. Clin Infect Dis 2017; 65: 1984-1991.

50. Haraszthy VI, Gerber D, Clark B, Moses P, Parker C, Sreenivasan PK et al. Characterization and prevalence of Solobacterium moorei associated with oral halitosis. J Breath Res 2008; 2: 017002.

51. Vancauwenberghe F, Dadamio J, Laleman I, Van Tornout M, Teughels W, Coucke W et al. The role of Solobacterium moorei in oral malodour. J Breath Res 2013; 7: 046006.

52. Rolph HJ, Lennon A, Riggio MP, Saunders WP, Mackenzie D, Coldero L et al. Molecular Identification of Microorganisms from Endodontic Infections. J Clin Microbiol 2001; 39: 3282-3289.

53. Rôças IN, Hülsmann M, Siqueira JF. Microorganisms in Root Canal-treated Teeth from a German Population. J Endod 2008; 34: 926-931.

54. Yang R, Yu T, Liu D, Shi S, Zhou Y. Hydrogen sulfide promotes immunomodulation of gingiva-derived mesenchymal stem cells via the Fas/FasL coupling pathway. Stem Cell Res Ther 2018; 9: 62.

55. Shouval R, Eshel A, Dubovski B, Kuperman AA, Danylesko I, Fein JA et al. Patterns of salivary microbiota injury and oral mucositis in recipients of allogeneic hematopoietic stem cell transplantation. Blood Adv 2020; 4: 2912-2917.

56. Whangbo J, Ritz J, Bhatt A. Antibiotic-mediated modification of the intestinal microbiome in allogeneic hematopoietic stem cell transplantation. Bone Marrow Transplant 2017; 52: 183-190.

\section{Tables}

Due to technical limitations, table 1 is only available as a download in the Supplemental Files section. 

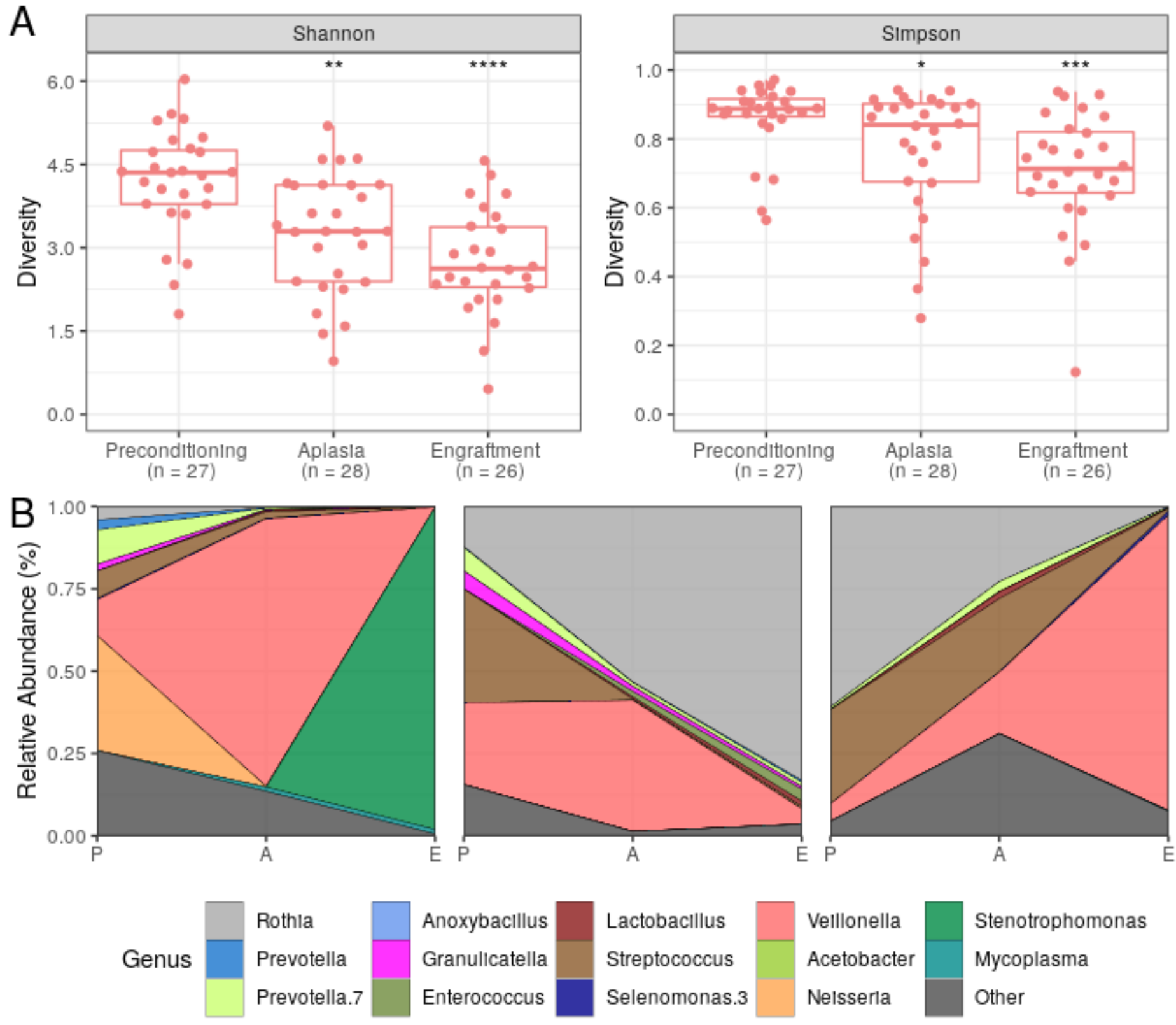

\section{Figure 1}

Bacterial diversity within the oral mucosa decreases during allo-HSCT. (A) Oral microbiota (OM) bacterial diversity boxplot at preconditioning $(n=27)$, aplasia $(n=28)$, and engraftment $(n=26)$ as measured by either Shannon index (left panel) or Simpson index (right panel). Mann-Whitney U tests were used with the preconditioning collection as the reference for comparisons. The boxes highlight the median values and cover the 25th and 75th percentiles, with whiskers extending to the more extreme value within 1.5 times the length of the box. Outliers are represented explicitly. Asterisks represent statistical significance: $\star, P<0.05 ; * \star, P<0.01 ; * \star \star, P<0.001 ; * \star \star \star, P<0.0001$. (B) OM genera relative abundance composition across transplantation phases for three representative patients showing the decrease in bacterial diversity. Only genera showing relative abundance $\geq 30 \%$ in at least one study sample or relative 
abundance $\geq 5 \%$ in at least $25 \%$ of study samples are shown. P, preconditioning; $A$, aplasia; $E$, engraftment.

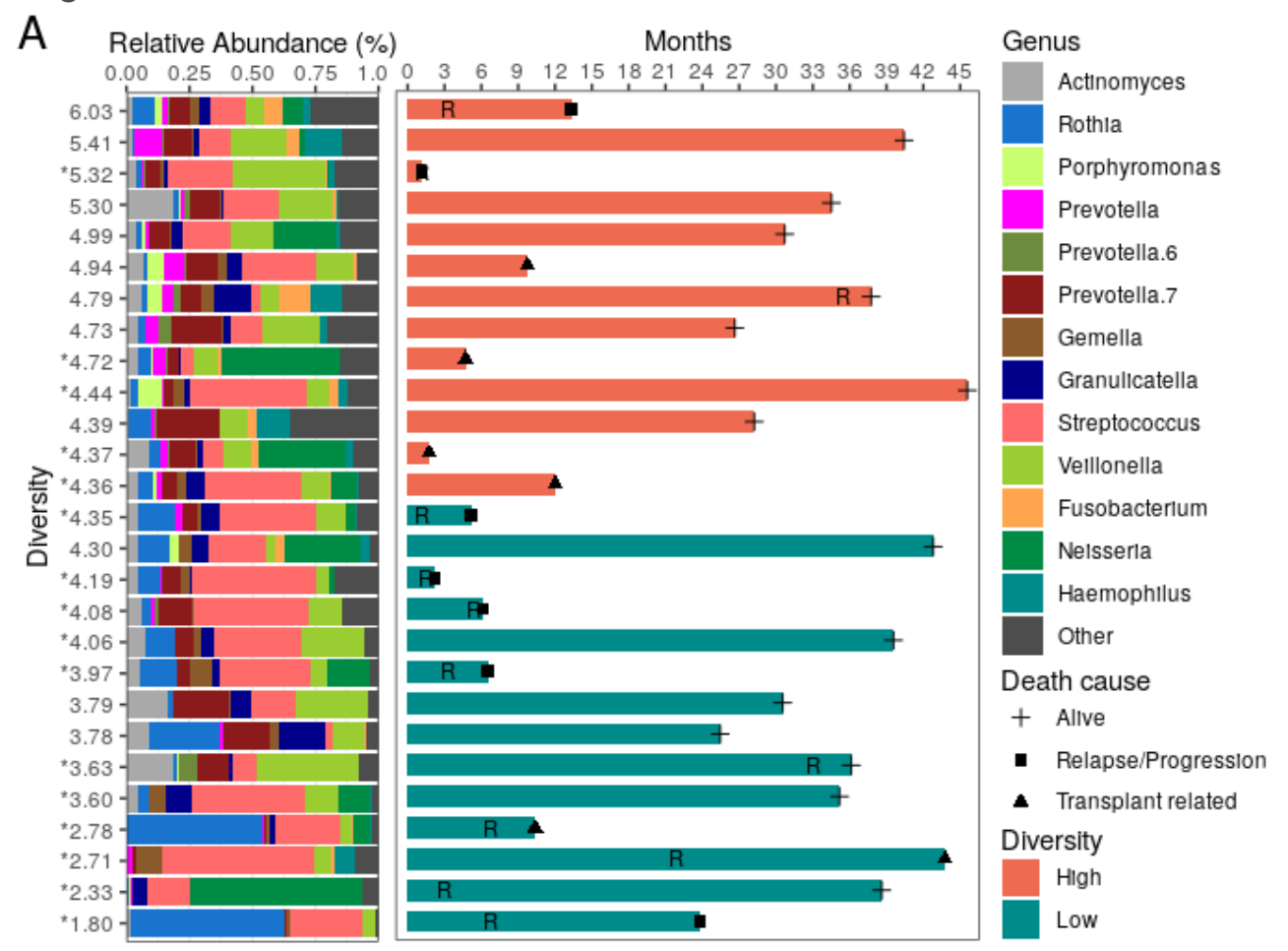

\section{B Diversity at Preconditioning}

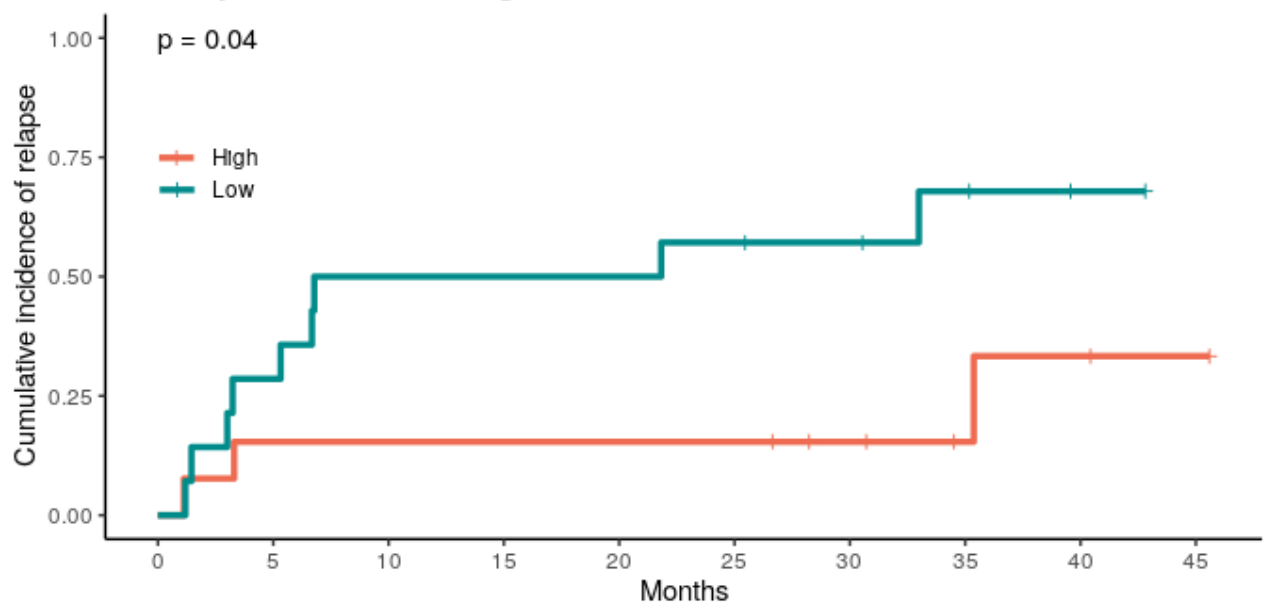

Number at risk

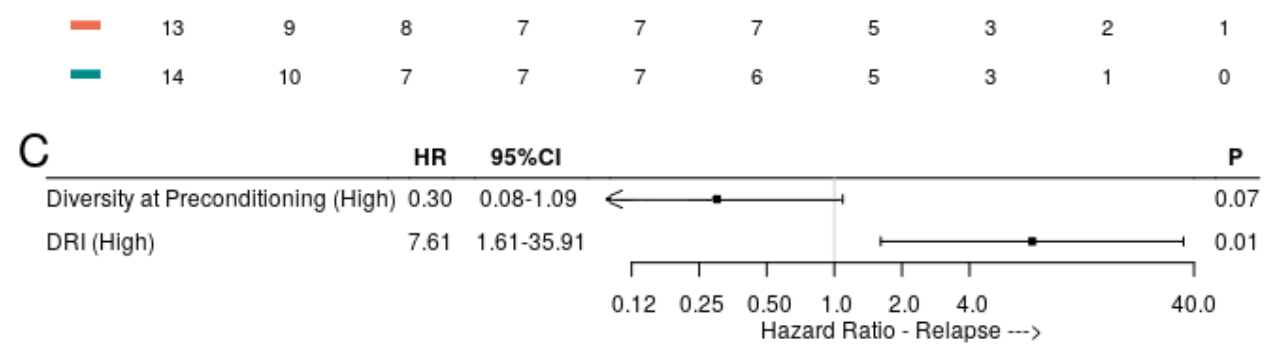

\section{Figure 2}

Oral microbiota bacterial dominance and bacterial diversity at preconditioning increased the risk of relapse in patients who underwent allo-HSCT. (A) Oral microbiota (OM) composition and diversity at preconditioning and the respective transplant course in each patient $(n=27)$. Patients are sorted based 
on descending Shannon diversity index, with the measures shown in the left subplot y-axis. The asterisk in the Shannon index indicates patients with at least one dominant (relative abundance $>30 \%$ ) genus at preconditioning. Only genera showing relative abundance $\geq 30 \%$ in at least one preconditioning sample or relative abundance $\geq 5 \%$ in at least $10 \%$ of preconditioning samples are shown. Relevant outcomes (relapse and death) after infusion (aplasia) are shown in a timeline (in months) subplot (right). The plus sign represents censoring. $R$, relapse. (B) Cumulative incidence of relapse with patients $(n=27)$ stratified by OM bacterial diversity at preconditioning (high versus low). (C) The DRI-adjusted hazard ratio for the association of OM bacterial diversity at preconditioning and relapse $(n=27)$.

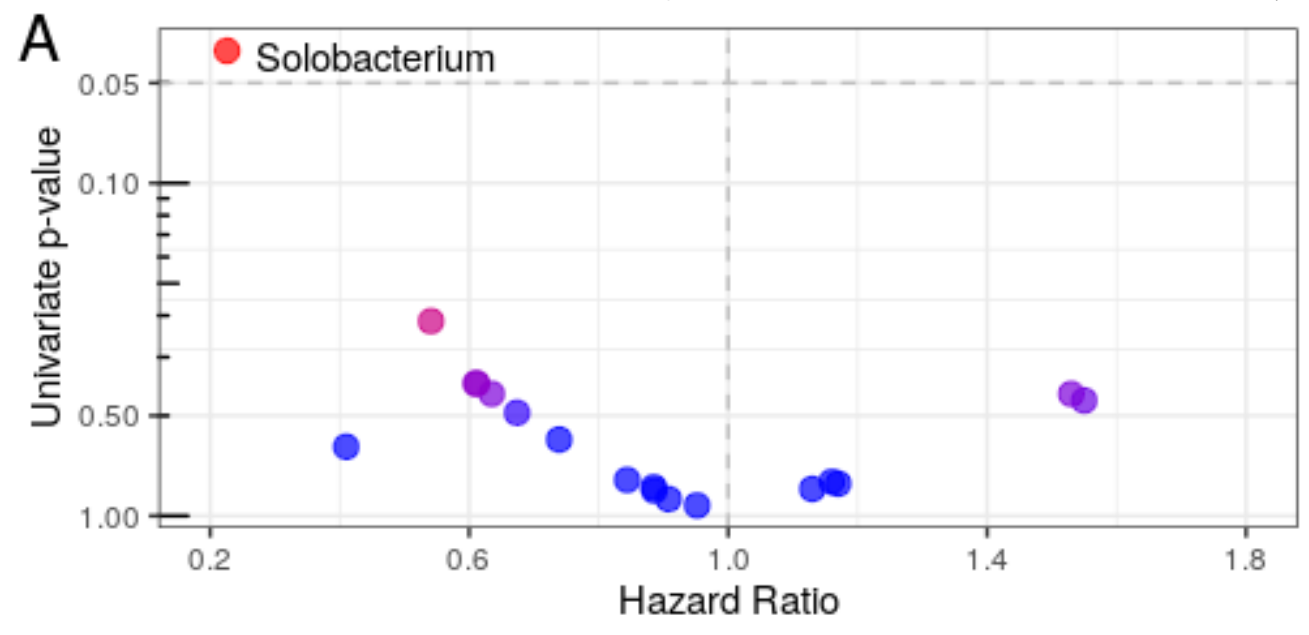

\section{B Solobacterium}

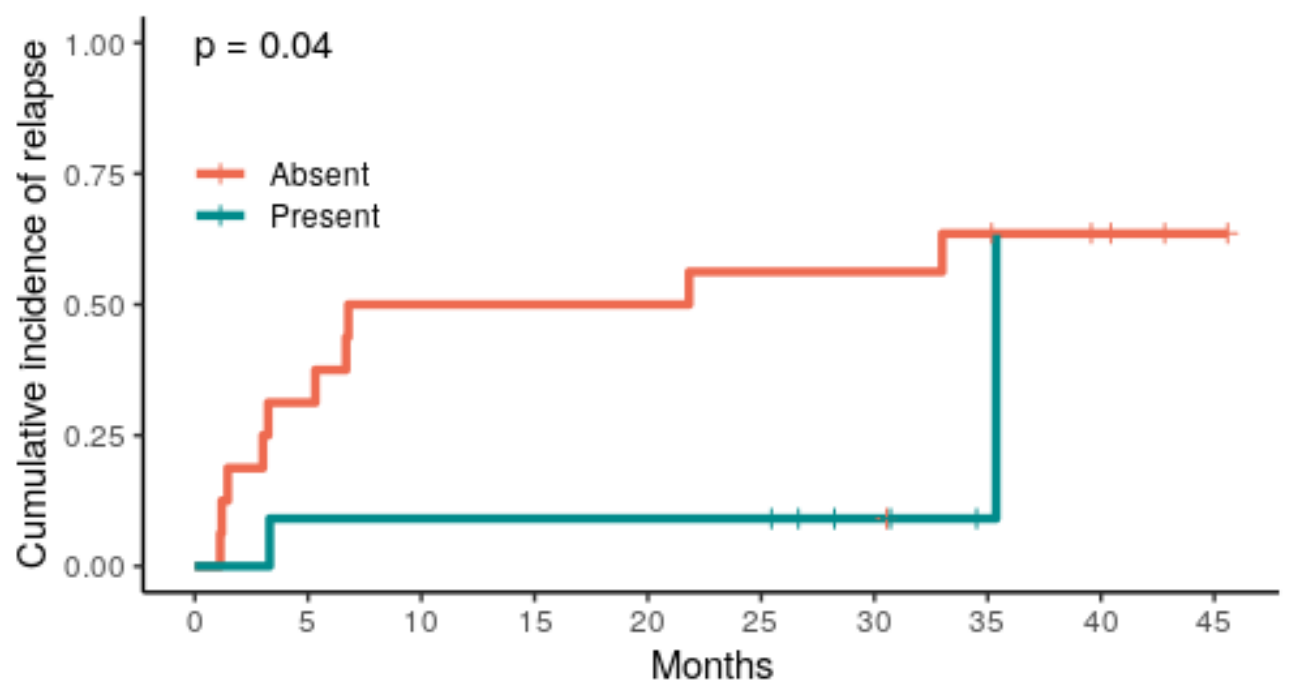

Number at risk

$\begin{array}{rrrrrrrrrr}16 & 11 & 8 & 8 & 8 & 7 & 7 & 5 & 3 & 1 \\ 11 & 8 & 7 & 6 & 6 & 6 & 3 & 1 & 0 & 0\end{array}$

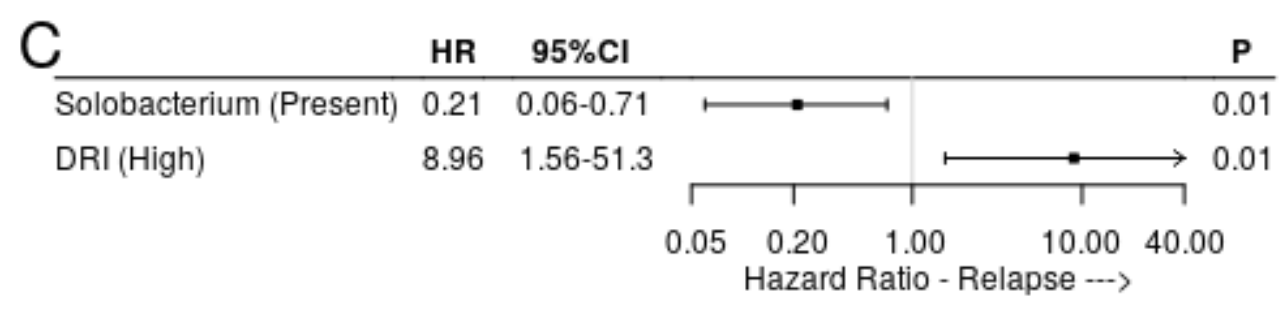




\section{Figure 3}

Association of any genus dominance with relapse, progression-free survival, and overall survival. (A) Cumulative incidence of relapse with patients $(n=27)$ stratified by any genus dominance at preconditioning. (B) Progression-free survival (PFS) with patients $(n=27)$ stratified by any genus dominance at preconditioning. (C) Overall survival (OS) with patients $(n=27)$ stratified by any genus dominance at preconditioning. (D) The DRl-adjusted hazard ratio for the association of dominance (relative abundance $>30 \%$ ) of any genus at preconditioning and relapse $(n=27)$. (E) The DRl-adjusted hazard ratio for the association of dominance (relative abundance $>30 \%$ ) of any genus at preconditioning and PFS $(n=27)$. (F) The DRI-adjusted hazard ratio for the association of dominance (relative abundance $>30 \%$ ) of any genus at preconditioning and OS $(n=27)$.
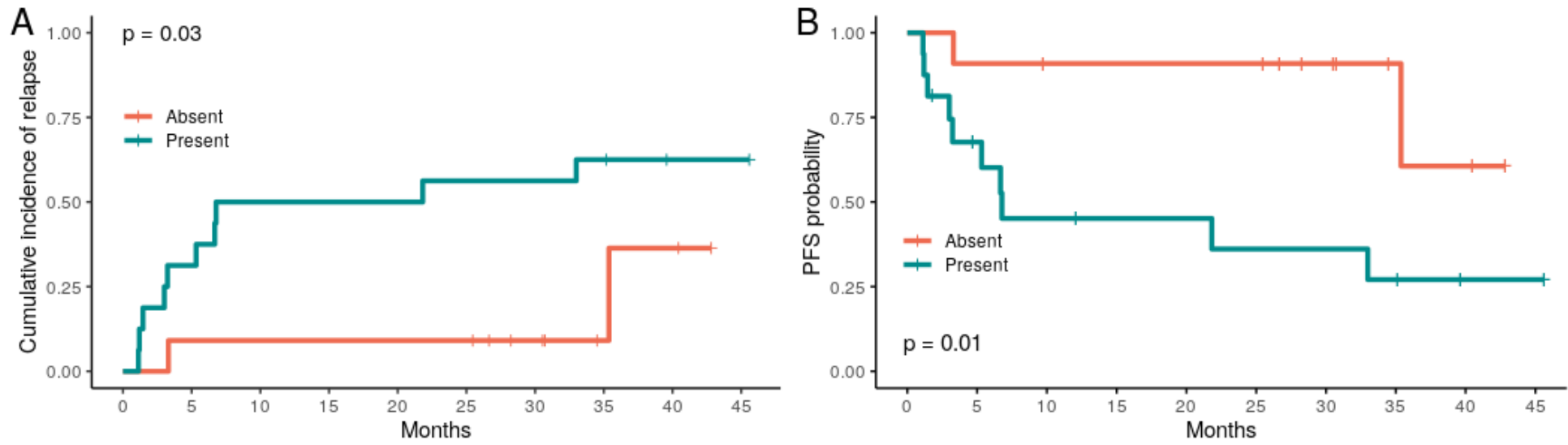

Number at risk

Number at risk
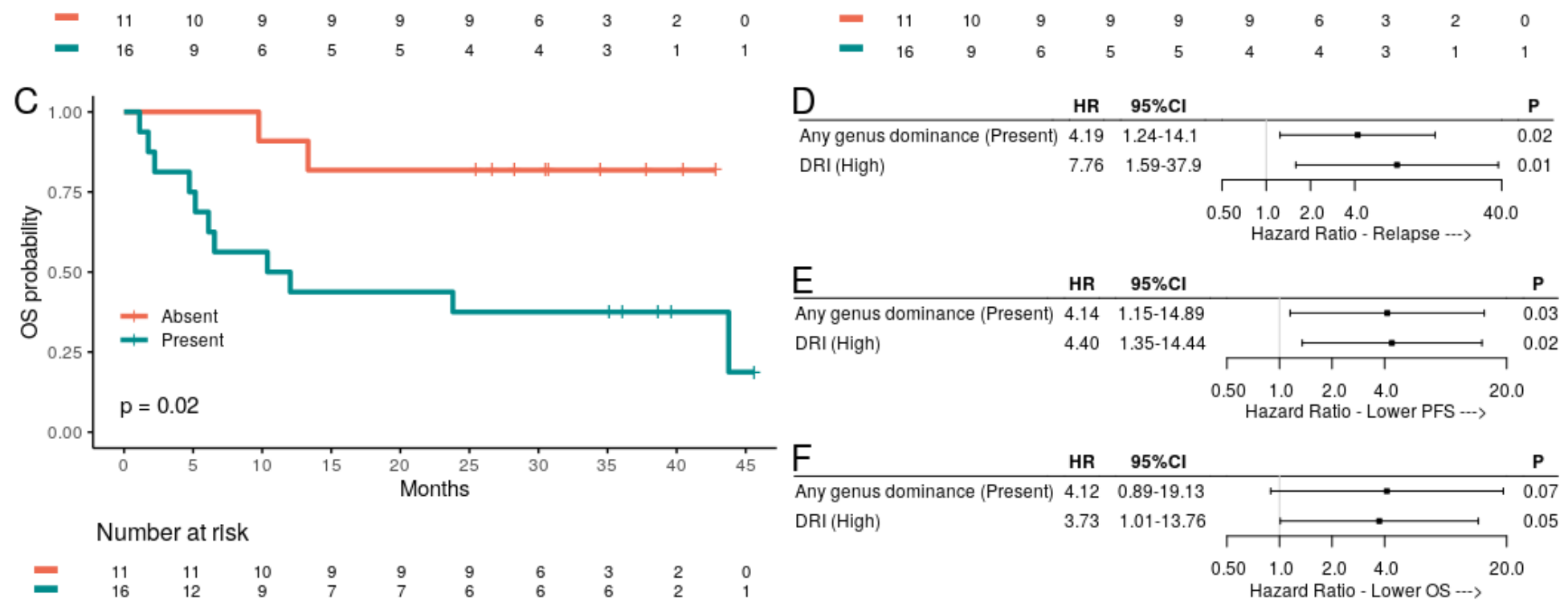

\section{Figure 4}

Solobacterium absence at preconditioning was associated with an increased risk of relapse in patients who underwent allo-HSCT. (A) Volcano plot for the univariate competing risk analysis of the association of relapse with the presence of specific genera at preconditioning (P value versus hazard ratio). The Solobacterium data point is indicated as it was the only genus significantly associated with relapse $(\mathrm{P}<$ 
0.05). (B) Cumulative incidence of relapse with patients $(n=27)$ stratified by Solobacterium presence. (C) The DRI-adjusted hazard ratio for the association of Solobacterium presence at preconditioning and relapse $(n=27)$.

\section{Supplementary Files}

This is a list of supplementary files associated with this preprint. Click to download.

- table1.xIsx

- SUPPLEMENTARYMATERIAL.docx 